\title{
BASIC FUNCTIONAL ANALYSIS PUZZLES OF SPECTRAL FLOW
}

\author{
B. BOOSS-BAVNBEK
}

(Received 6 August 2010; accepted 16 December 2010)

Communicated by S. Rosenberg

Dedicated to Alan Carey, on the occasion of his 60th birthday

\begin{abstract}
We explain an array of basic functional analysis puzzles on the way to general spectral flow formulae and indicate a direction of future topological research for dealing with these puzzles.
\end{abstract}

2010 Mathematics subject classification: primary 58J37; secondary 35J67, 57Q20, 58J32, 58J50.

Keywords and phrases: Dirac operator, perturbations, spectral flow.

\section{Introduction}

Over the last decades, substantial progress has been achieved in analytic approaches to spectral flow in various geometric, topological, and operator algebra settings. For a taste of some recent results see, for example, Benameur et al. [2].

Each new approach and each new context displays new and surprising features, radically new difficulties to be overcome, and astonishing aspects of the new results. How can it be that seemingly small changes of the setting require different methods and types of assumptions and yield radically different results?

One explanation can be found in the array of basic functional analysis puzzles connected with the concept of the spectral flow and its calculation. In Section 2, we fix the notation and recall the most elementary spectral flow formula, relating the symmetric category of curves of self-adjoint Fredholm operators in separable Hilbert space with the skew-symmetric category of symplectic functional analysis. Moreover, we shall point to peculiar functional analytic properties of geometrically defined operators like Dirac-type operators. We explain which of these properties can be regained for general elliptic operators and how. In Section 3, we present our list of basic functional analysis puzzles on the way to general spectral flow formulae. In Section 4, we indicate a promising further direction to deal with these 'puzzles'.

(C) 2011 Australian Mathematical Publishing Association Inc. 1446-7887/2011 \$16.00 


\section{The model case of the functional analytic approach}

To investigate spectral properties of geometrically defined differential operators like the Laplacian and the Dirac operator on manifolds with boundary and on partitioned manifolds, one has to draw on a variety of tools.

2.1. The von Neumann approach. Some common deep functional analytic roots of these formulae have been revealed by Furutani and the author in [4], emphasizing the role of the Cauchy data spaces. More precisely, in the von Neumann-KreunVishik-Birman tradition, one is given a complex separable Hilbert space $\mathcal{H}$ and a closed symmetric operator $A$. One defines the symplectic Hilbert space of abstract boundary values by $\beta(A):=\operatorname{dom}\left(A^{*}\right) / \operatorname{dom}(A)$ with naturally induced inner product $\langle[x],[y]\rangle$ and symplectic form $\omega([x],[y])$ and the natural Cauchy data space $\mathrm{CD}(A):=\left\{[x] \mid x \in \operatorname{ker} A^{*}\right\}$. One has a canonical correspondence between all selfadjoint extensions $A_{D}$ of $A$ with domain $D$ and the Lagrangian subspaces $[D] \subset \beta(A)$. In this framework, for example, if $A_{D}$ is a self-adjoint Fredholm extension and $\left\{C_{t}\right\}$ a continuous curve in $\mathcal{B}(\mathcal{H})$ with $\operatorname{ker}\left(A^{*}+C_{t}+s\right) \cap \operatorname{dom}(A)=\{0\}$ for small $|s|$ (weak inner uniform continuation property, abbreviated to UCP), one obtains that $\left\{\mathrm{CD}\left(A+C_{t}\right),[D]\right\}$ is a continuous curve of Fredholm pairs of Lagrangians and $\operatorname{SF}\left\{\left(A+C_{t}\right)_{D}\right\}=\operatorname{MAS}\left\{\mathrm{CD}\left(A+C_{t}\right),[D]\right\}$, relating the spectral flow of a selfadjoint Fredholm operator under bounded variation with the Maslov index of the corresponding curve of Lagrangians in the abstract boundary space.

The strength of this functional analytic approach turns up when dealing with systems of ordinary differential equations on the interval, generalizing the classical Morse index theorem for geodesics on Riemannian manifolds to sub-Riemannian manifolds. It recovers the Floer-Yoshida-Nicolaescu splitting results for the spectral flow of curves of Dirac operators on partitioned manifolds (that is, the family version of the Bojarski conjecture), and it provides a basic functional analytic model for quantization and tunneling, relating spectral and symplectic invariants.

2.2. The challenge of varying domain. How can we transgress the limitations of the general functional analytic approach? What if we do not keep the domain fixed under variation; nor restrict ourselves to bounded (that is, zero order) perturbations; nor confine the applicability to ordinary differential equations or Dirac-type operators with constant coefficients in the normal direction (product case) close to the boundary? A series of recent papers took up the challenge of perturbed Dirac operators and general linear elliptic differential operators; investigated weak inner UCP; established the existence of self-adjoint Fredholm extensions; admitted variation of domain and skew boundaries; and investigated uniform structures and continuous perturbations; see, for example, Axelsson et al. [1]; joint work of the author with Chen, Lesch, and Zhu in [3, 5, 7, 10]; Eichhorn [14]; and Gesztesy et al. [15]. We conclude that the 'natural' (von Neumann) approach is insufficient, and more analysis (for example, splitting the coefficients near the boundary and pseudodifferential calculus) is needed. 
2.3. Seeley's Calderón projection and Dirac operator folklore. Let $M$ be a smooth compact Riemannian manifold with boundary $\Sigma, E, F$ be Hermitian vector bundles over $M$, and $A: C^{\infty}(M, E) \rightarrow C^{\infty}(M, F)$ be an elliptic differential operator (of first order). Recall that $\rho: L_{s}^{2}(M, E) \rightarrow L_{s-1 / 2}^{2}\left(\Sigma, E_{\Sigma}\right)$ for $s>1 / 2$ is extendable to $\mathcal{D}_{\max }(A)$. Then the classical definition of the Cauchy data space $N_{+}^{0}(A)$ of $A$ is the closure of $\left\{\rho u \mid A u=0\right.$ in $\left.M \backslash \Sigma, u \in C^{\infty}(M, E)\right\}$ in $L^{2}\left(\Sigma, E_{\Sigma}\right)$. Seeley [22, 23] proved that this Cauchy data space can be obtained as the range of a pseudodifferential projection. The basic ingredients for Seeley's result have been the construction of an invertible extension $\widetilde{A}$ of $A$ over a closed manifold $\widetilde{M}$ by extending $A$ to a collar, then doubling and applying symbolic calculus and UCP management. As a result, he obtained a Poisson operator $K_{ \pm}:= \pm r^{ \pm} \widetilde{A}^{-1} \rho^{*} J(0)$, where $J(0)=\sigma(A)(\cdot, v) \in$ End $\left(E_{\Sigma}\right)$ denotes the principal symbol of $A$ in the normal direction at the boundary. He showed that the operator $C_{ \pm}:=\rho K_{ \pm}$is a pseudodifferential projection onto $N_{+}^{0}(A)$ and called it the Calderón projection.

It was shown by Wojciechowski and the author [9, Chs. 9 and 12] that Seeley's construction is canonical (that is, natural, explicit, transparent, and free of choices) for Dirac-type operators when the metric structures are products close to the boundary. As a consequence, we obtained the Lagrangian property of the Cauchy data space. The reason is that for such operators the invertible extension $\widetilde{A}$ can be explicitly defined on the very closed double $\widetilde{M}$ of $M$, without inserting an additional collar near the boundary and not involving any other choices. As a consequence, the Cauchy data spaces and the Calderón projection vary continuously under smooth deformation of the data defining the Dirac operator, as was proved by Lesch, Phillips, and the author in [6].

These results for Dirac-type operators can be traced back to the 'Dirac operator folklore': (i) weak inner UCP, that is, $\operatorname{ker} A \cap \operatorname{dom}\left(A_{\min }\right)=\{0\}$ with $\operatorname{dom}\left(A_{\min }\right)=$ $L_{1, \text { comp }}^{2}(M, E)$; (ii) a symmetric principal symbol of the tangential operator $B$ in the decomposition $A=J_{0}\left(\partial_{x}+B\right)$, where $x$ denotes the inner normal variable; and (iii) a precise invertible double. From that alone, one can derive the transparent definition of the Calderón projection, the Lagrangian property of the Cauchy data space, the existence of a self-adjoint Fredholm extension given by a regular pseudodifferential boundary condition, the cobordism theorem, and the continuous dependence of input data.

One may wonder how special operators of Dirac type are compared to arbitrary linear first-order elliptic differential operators. The short answer is that property (i) may be lost but is indispensable, hence must be assumed. Property (ii) implies property (i) (if it is valid for the tangential operators on arbitrary hypersurfaces), but otherwise it is dispensable (for details, see Section 3 below). Property (iii) can be maintained by replacing Seeley's classical construction by a new construction, inspired by Himpel et al. [16] and worked out in [7].

2.4. The invertible double, revisited. We summarize the new construction. First, we bring a given general elliptic differential operator of first order in product form $A=J\left(\partial_{x}+B\right)$ close to the boundary by suitable choice of the metric. Here, $J$ and $B$ 
vary with the normal variable $x$. Note that in dropping the geometric Dirac operator context, the metric structures need no longer be fixed.

We obtain a canonical new invertible double $\tilde{A}_{T}$ with

$$
\operatorname{dom}\left(\tilde{A}_{T}\right):=\left\{\left(\begin{array}{l}
e \\
f
\end{array}\right) \in L_{1}^{2}(M, E \oplus F) \mid \varrho f=T \varrho e\right\},
$$

where

$$
\tilde{A}: C^{\infty}(M, E \oplus F) \stackrel{A \oplus\left(-A^{t}\right)}{\longrightarrow} C^{\infty}(M, F \oplus E)
$$

and $T \in \operatorname{Hom}\left(\Sigma,\left.E\right|_{\Sigma},\left.F\right|_{\Sigma}\right)$ is an invertible bundle map with $J_{0}^{*} T$ positive definite. Then $\tilde{A}_{T}$ is a Fredholm operator with compact resolvent, $\operatorname{ker} \tilde{A}_{T}=Z_{+, 0} \oplus Z_{-, 0}$ and coker $\tilde{A}_{T} \simeq Z_{-, 0} \oplus Z_{+, 0}, \quad$ where $Z_{+, 0}:=\left\{f \in L_{1}^{2}(M, E) \mid A f=0, \varrho f=0\right\}$ and $Z_{-, 0}$ denotes the corresponding kernel of $A^{t}$. For the most part of our work, we pick $T:=\left(J_{0}^{t}\right)^{-1}$. Denoting the pseudoinverse of $\tilde{A}_{T}$ by $\tilde{G}$, we define Poisson operators

$$
K_{ \pm}:= \pm r^{ \pm} \tilde{G} \varrho^{*} J_{0}: L_{s}^{2}(\Sigma, E) \rightarrow L_{s+\frac{1}{2}}^{2}(M, E)\left(L_{s+\frac{1}{2}}^{2}(M, F)\right)
$$

and Calderón operators $C_{+}:=\varrho_{+} K_{+}, C_{-}:=T^{-1} \varrho_{-} K_{-}$. We obtain that $C_{ \pm}$are projections with $C_{+}+C_{-}=I$ and $C_{+}\left(L^{2}\right)=N_{+}^{0}, C_{-}\left(L^{2}\right)=T^{-1} N_{-}^{0}$.

The most delicate part of the new construction is the investigation of the mapping properties of the pseudoinverse $\widetilde{G}$, the Poisson operators $K_{ \pm}$, and the Calderón projection $C_{ \pm}$.

Our model operator is $A=J((\partial / \partial x)+B(x))+0$ order. From the ellipticity of $A$, we have that $i \xi+B(x)$ is invertible for real $\xi$ of sufficiently large numerical value (array of minimal growth). We put $Q_{+}(x):=(1 / 2 \pi i) \int_{\Gamma_{+}} e^{-x \lambda}(\lambda-B(0))^{-1} d \lambda$, a family of sectorial projections, where $\Gamma_{+}$is a contour which encircles the eigenvalues of $B(0)$ in the right half plane. We notice that $Q_{+}(x)$ corresponds to $e^{-x B(0)} 1_{[0, \infty)}(B(0))$ if $B(0)=B(0)^{*}$. We had to display a delicate balance on a knife edge between general operator theory and pseudodifferential calculus when we realized that a priori $Q_{+}(x)=O(\log x), x \rightarrow 0+$, hence $P_{+}:=Q_{+}(0)$ is possibly unbounded. Within the pseudodifferential calculus, it follows, however, from Burak [11], and a result by Wodzicki explained in Ponge [21, Proposition 4.1], that $P_{+}:=Q_{+}(0)$ is a bounded pseudodifferential projection. A posteriori, we obtain $Q_{+}(x) \rightarrow P_{+}$ strong, $x \rightarrow 0+$.

Another hopefully useful concept introduced in [7] is the approximative Poisson operator $R: C^{\infty}(\partial M, E) \longrightarrow C^{\infty}\left(\mathbb{R}_{+} \times \partial M, E \oplus F\right)$ with $R \xi(x):=\varphi(x)\left(\begin{array}{c}Q_{+}(x) \xi \\ T Q_{-}(x) \xi\end{array}\right)$, where $\varphi$ is a suitable cut-off function at 0 . One finds that $R=\tilde{A}_{T}^{-1} \varrho^{*}$ and a regularizing remainder. That permits us to analyze the mapping property of $R: L_{S}^{2}(\partial M, E) \rightarrow$ $L_{s^{\prime}}^{2}\left(\mathbb{R}_{+} \times \partial M, E \oplus F\right)$ in dependence on $A$.

2.5. A recent result on sectorial projections. Regarding uniform structures, it turns out that $C_{+}(A)-P_{+}(B(0))$ is a pseudodifferential operator of order -1 and 
that $A \mapsto C_{+}(A)$ is as regular as $A \mapsto P_{+}(B(0))$ under the condition $\operatorname{dim} Z_{0}(A)$, $\operatorname{dim} Z_{0}\left(A^{t}\right)=$ const. Now, [3, Theorem 1.1] proves that the sectorial projections of elliptic differential operators on closed manifolds depend continuously on the initial operator in the natural Fréchet topology, if there exist suitable spectral cuttings for the principal symbol (like no purely imaginary eigenvalues of the principal symbol, which is exactly satisfied for the tangential operator $B(0)$ of any elliptic operator $A$ over a compact smooth manifold with boundary). Consequently, the Calderón projection $C_{+}(A)$ varies continuously in the operator norm of bounded operators on $L^{2}(\Sigma)$, if the coefficients of $A$ and all its derivatives vary continuously. Moreover, one obtains that $(A, P) \mapsto A_{P}$ is continuous in graph topology, if $P$ runs in the space of 'regular' boundary conditions.

Further applications for $A=A^{t}$ are that the Cauchy data space is Lagrangian in the Hermitian symplectic Hilbert space $\left(L^{2}(\partial M, E),\langle\cdot, J(0) \cdot\rangle\right)$; the existence of a self-adjoint Fredholm extension $A_{C_{ \pm}}$(for suitable choice of $T$ ); and the cobordism invariance of the index for arbitrary symmetric elliptic differential operators on closed manifolds: sign $i J(0)$ vanishes on $\bigoplus_{\lambda \text { imaginary }} \operatorname{ker}(B(0)-\lambda)^{N}, N \gg 0$.

\section{A personal choice of functional analysis puzzles}

From the preceding summary, we can extract an array of functional analytic puzzles on the way to general spectral flow formulae.

3.1. Geometrically defined versus general coefficients. In applications, there is a decisive difference between ad hoc models and models based on first principles, as pointed out, for example, by Manin [20]. Ad hoc models are based on fancied hypotheses about the interrelation between different features and on estimates of the rates and other coefficients. Mathematically speaking, they require general coefficients. On the contrary, equations and coefficients in theoretically based models have a direct meaning, for example, when derived from minimal principles. Often, to exploit this meaning one better restricts the consideration to geometrically defined operators, instead of striving for the goal of 'highest generality'. Clearly, for gaining mathematical insight both approaches have their merits and yield their own way of transparency. In the example presented above in Section 2, the Dirac case yields a simple construction of the invertible double while the general approach yields a list of universal essentials for getting through.

3.2. Fixed operator versus deformation curve. Addressing curves instead of single points is as old as celestial mechanics and variational calculus. To embed such questions in a systematic way into a family setting of deformations is not a new idea; it goes back to Lagrange's second letter (in Latin) to Euler regarding the derivation of what is now called the Euler-Lagrange equation [19]. Following Lagrange, it seems a tenet of mathematics in our time to address deformation questions at the first place. As a rule it turned out, for example, in index theory that family versions are more demanding than single-operator formulae. In contractible spaces the situation is 
different when, for example, the spectral flow of a curve solely depends on the end points. Then, like in Lagrange's idea, the embedding of a problem into a deformation curve may facilitate the treatment and not complicate it.

3.3. Bounded versus unbounded operators. With some right, we may forget about that distinction when working with an elliptic operator $A$ (say symmetric and of order one) on a closed manifold $M$. Then there is no difference between minimal and maximal domains. They are always equal to the Sobolev space $L_{1}^{2}(M)$. Moreover, in that case the Riesz transform $A \mapsto A\left(I+A^{2}\right)^{-1 / 2}$ yields a bounded operator in $L^{2}(M)$ and is continuous in suitable operator norms, see [9, Ch. 17]. The situation is much more blurred for elliptic operators on manifolds with boundary. There, the general functional analysis picture has strongly counterintuitive traits.

Let $\mathcal{C} \mathcal{F}(H)$ denote the space of closed (not necessarily bounded) Fredholm operators in a fixed complex separable Hilbert space $H$ and let $\mathcal{C} \mathcal{F}^{\mathrm{sa}}(H)$ denote the subspace of self-adjoint elements. For index theory, Cordes and Labrousse [12] have shown that the index is constant on the connected components of $\mathcal{C F}(H)$ and yields a bijection between the integers and the connected components. For the spectral flow, quite a different result was proved in [6]: while the space of bounded self-adjoint Fredholm operators decomposes into three connected components (the contractible spaces of essentially positive, respectively essentially negative, operators and the nontrivial component with homotopy type of Bott periodicity), the space $\mathcal{C} \mathcal{F}^{\mathrm{sa}}(H)$ is connected and its homotopy type is not fully revealed. Moreover, equipping the space $\mathcal{C} \mathcal{F}^{\text {sa }}(H)$ with the graph (gap) topology and the space of bounded operators with the operator norm, the Riesz transform is not continuous, as shown by a counterexample provided by B. Fuglede (for details, see [6, Example 2.14]).

3.4. Self-adjoint versus general. Motivated by the method of replacing a differential equation by difference equations, Hilbert and Courant [13] expected 'linear problems of mathematical physics which are correctly posed to behave like a system of $N$ linear algebraic equations in $N$ unknowns .... If for a correctly posed problem in linear differential equations the corresponding homogeneous problem possesses only the trivial solution zero, then a uniquely determined solution of the general inhomogeneous system exists. However, if the homogeneous problem has a nontrivial solution, the solvability of the inhomogeneous system requires the fulfillment of certain additional conditions.' This is the heuristic principle, which Hilbert and Courant saw in the Fredholm alternative. G. Hellwig in the real setting and I. N. Vekua in the complex setting (both nicely explained recently by Kalf [17]) disproved it in 1952. Independently of each other, they discovered symmetric differential operators on the disc with nonself-adjoint boundary condition where the Fredholm alternative fails.

From the chiral splitting of Dirac-type operators we have learnt that self-adjoint and nonself-adjoint problems can be related to each other. One instance is the Cobordism theorem for two linear elliptic, not necessarily symmetric operators on 
closed manifolds which appear as components of the tangential operator for a selfadjoint boundary problem [9, Corollary 21.6].

It is remarkable how easy it is to apply the spectral theorem to prove the continuous dependence of spectral projections outside a spectral cut for symmetric elliptic differential operators on closed manifolds (see [7, Proposition 7.15]) and how elaborate the arguments become for proving a similar result without symmetry assumptions (see [3]).

3.5. Functional analysis versus pseudodifferential analysis. The investigation of the mapping properties for constructing sectorial and Calderón projections from elliptic operators yields a treasure of situations where claims can be formulated in general functional analytic terms but be proved only by advanced pseudodifferential analysis. As examples, see the preceding discussion of the boundedness of the sectorial projection $P_{+}(B(0))$; the coincidence of the mapping property of $B(0) \mapsto$ $P_{+}(B(0))$ and $A \mapsto C_{+}(A)$; and the mentioned recent delicate proof of the continuous dependence of $P_{+}(B(0))$ on $B(0)$.

3.6. Strong symplectic versus weak symplectic. From classical mechanics and the usual treatment of Dirac operators, we are accustomed to strong symplectic structures, that is, we assume that the symplectic form $\omega$ can be written as a scalar product $\omega(x, y)=\langle J x, y\rangle$ with bounded invertible (that is, also the inverse is bounded) generator operator $J$. On a smooth compact manifold $M$ with boundary $\Sigma$, any elliptic operator $A$ (say of order one and symmetric) induces strong symplectic structures on the von Neumann boundary value space $\beta$ defined above in Section 2 and on $L^{2}(\Sigma)$ with $J$ defined by the principal symbol of $A$ over $\Sigma$ in inner normal direction. Formally, in the same way, we obtain a symplectic structure for the Sobolev space $L_{1 / 2}^{2}(\Sigma)$ where all the boundary values of the domain of the extensions of $A$ are placed by Sobolev restriction. However, if $\operatorname{dim} \Sigma \geq 1$, that structure is no longer strong but becomes weak, see [10, Section 2, Remark]. In weak symplectic analysis, we do not know whether the space of Lagrangian subspaces is contractible; whether the homotopy of the space of Fredholm pairs of Lagrangian subspaces is of Bott periodicity; nor whether there exist Fredholm pairs of Lagrangian subspaces with negative index, see [10, Section 2.3].

3.7. Weak inner UCP? For operators of Dirac type, the weak unique continuation property can be obtained in two different ways, either by exploiting the fact that the principal symbol of the Dirac Laplacian is in diagonal form and real or by exploiting the fact that the principal symbol of the tangential operators are symmetric for all hypersurfaces, see [9, Ch. 8] for details or [5, Theorem 1.3] for outlines and references. In contrast to the usual unique continuation property for elements belonging to the kernel of an elliptic operator, the property weak inner UCP, discussed above in Section 2, is purely functional analytic. As an immediate consequence, Zhu and the author obtained the local stability of weak inner UCP; for references and wider ramifications, see [5, Section 4]. The stability of weak (global) UCP was obtained by 
the author and Marcolli and Wang [8] for mild nonlinear perturbations of the Dirac operator, motivated by Seiberg-Witten theory.

We shall not elaborate on the many other puzzles. For instance, one may wonder about the functional analytic roots of the noted differences between homotopy invariance, valid for index and spectral flow in suitable setting, and solely spectral invariance of $\eta$-invariant and $\zeta$-function regularized determinants. Another puzzle, not addressed here, are the differences and relations between the desuspension character of spectral flow formulae going a dimension down (mostly rather delicate from an analysis point of view) and the suspension character of rather different spectral flow formulae, going a dimension up (and often more easily accessible).

\section{How to deal with these puzzles?}

History of mathematics (and of the sciences, as well) provides ample evidence of changes between periods of expansion (diversification) and periods of consolidation (establishing deep, principal interrelations). A famous case is how the ideas of R. Bott, F. Hirzebruch, I. M. Singer, and M. F. Atiyah (and followers) lead to the identification of Fredholm operators and index problems in wide fields of geometry and a corresponding unprecedented interconnection between topology, geometry, functional analysis, partial differential equations, dynamical systems, number theory, and mathematical physics. Similarly, one may expect that the avalanche of new results on spectral invariants of operator curves, though pointing in many seemingly unrelated directions, will help to single out one or two key concepts for dealing with the listed (and supplementary) 'puzzles' around spectral flow.

To overcome, or better to make maximal use of, the vast amount of inspiring, but spread calculations, it will not be easy to single out a specific direction of dealing with all the puzzles in one round. One candidate for such a unifying approach is the concentration on the homotopy type of the operator spaces involved. That was the starting point of my joint work with Wojciechowski; see, for example [9, Chs. 15-17]. The task is easy to formulate: look for the involved subspaces of unitary operators and check whether Bott periodicity is maintained, respectively determine deviations in homotopy type, and do it both in general functional analysis terms and in pseudodifferential operator terms. To me, the work, for example, by Kirk and Lesch [18, Sections 2 and 6] indicates that this program continues to be promising.

\section{Acknowledgements}

For the preceding considerations I must take the responsibility alone, assuming that most of the views were shared or will be shared by Alan Carey. The findings are also based on continuing discussions with students and colleagues. In particular, I am indebted to Kenro Furutani (Tokyo), Matthias Lesch (Bonn), Ryszard Nest (Copenhagen), John Phillips (Victoria), Chaofeng Zhu (Tianjin) and my late collaborator for decades Krzysztof Wojciechowski. 


\section{References}

[1] A. Axelsson, S. Keith and A. McIntosh, 'Quadratic estimates and functional calculi of perturbed Dirac operators', Invent. Math. 163 (2006), 455-497.

[2] M.-T. Benameur, A. L. Carey, J. Phillips, A. Rennie, F. A. Sukochev and K. P. Wojciechowski, 'An analytic approach to spectral flow in von Neumann algebras', in: Analysis, Geometry and Topology of Elliptic Operators, (eds. B. Booß-Bavnbek et al.) (World Scientific, Hackensack, NJ, 2006), pp. 297-352.

[3] B. Booß-Bavnbek, G. Chen, M. Lesch and C. Zhu, 'Perturbation of sectorial projections of elliptic pseudo-differential operators', Preprint, Roskilde and Tianjin 2010, 31 pages, arXiv:1101.0067v2 [math.SP].

[4] B. Booß-Bavnbek and K. Furutani, 'The Maslov index: a functional analytical definition and the spectral flow formula', Tokyo J. Math. 21 (1998), 1-34.

[5] B. Booß-Bavnbek and M. Lesch, 'The invertible double of elliptic operators', Lett. Math. Phys. 87(1-2) (2009), 19-46.

[6] B. Booß-Bavnbek, M. Lesch and J. Phillips, 'Unbounded Fredholm operators and spectral flow', Canad. J. Math. 57(2) (2005), 225-250.

[7] B. Booß-Bavnbek, M. Lesch and C. Zhu, 'The Calderón projection: new definition and applications', J. Geom. Phys. 59 (2009), 784-826.

[8] B. Booß-Bavnbek, M. Marcolli and B. L. Wang, 'Weak UCP and perturbed monopole equations', Internat. J. Math. 13(9) (2002), 987-1008.

[9] B. Booß-Bavnbek and K. P. Wojciechowski, Elliptic Boundary Problems for Dirac Operators (Birkhäuser, Basel, 1993).

[10] B. Booß-Bavnbek and C. Zhu, 'The Maslov index in weak symplectic functional analysis', Preprint, Roskilde 2010, 31 pages, see also arXiv:math/0406139v1 [math.DG] and arXiv:math/0504125v2 [math.DG].

[11] T. Burak, 'On spectral projections of elliptic operators', Ann. Sc. Norm. Super. Pisa (3) 24 (1970), 209-230.

[12] H. O. Cordes and J. P. Labrousse, 'The invariance of the index in the metric space of closed operators', J. Math. Mech. 12 (1963), 693-719.

[13] R. Courant and D. Hilbert, Methods of Mathematical Physics I and II (Interscience, New York, 1953/1962), translated from German.

[14] J. Eichhorn, 'Index theory for generalized Dirac operators on open manifolds', in: $C^{*}$-algebras and Elliptic Theory, Trends in Mathematics (Birkhäuser, Basel, 2006), pp. 73-128.

[15] F. Gesztesy, Y. Latushkin, K. A. Makarov, F. Sukochev and Y. Tomilov, 'The index formula and the spectral shift function for relatively trace class perturbations', Adv. Math. 227 (2011), 319-420.

[16] B. Himpel, P. Kirk and M. Lesch, 'Calderón projector for the Hessian of the Chern-Simons function on a 3-manifold with boundary', Proc. Lond. Math. Soc. (3) 89 (2004), 241-272.

[17] H. Kalf, 'Günter Hellwig-in memoriam', Sitzungsberichte der Berliner Mathematischen Gesellschaft, to appear.

[18] P. Kirk and M. Lesch, 'The $\eta$-invariant, Maslov index, and spectral flow for Dirac-type operators on manifolds with boundary', Forum Math. 16 (2004), 553-629.

[19] J. L. Lagrange, 'Letter to Euler 12 August 1755' (Latin), in: Oeuvres de Lagrange, 14 (eds. J. A. Serret, G. Darboux) (Gauthier-Villars, Paris, 1892), pp. 366-375.

[20] Y. Manin, 'Mathematical knowledge: internal, social and cultural aspects', in: Mathematics as Metaphor: Selected Essays of Yuri I. Manin with Foreword by Freeman J. Dyson (American Mathematical Society, Providence, RI, 2007), pp. 3-26.

[21] R. Ponge, 'Spectral asymmetry, zeta functions, and the noncommutative residue', Internat. J. Math. 17 (2006), 1065-1090. 
[22] R. T. Seeley, 'Singular integrals and boundary value problems', Amer. J. Math. 88 (1966), 781-809.

[23] R. T. Seeley, 'Topics in pseudo-differential operators', in: C.I.M.E., Conference on Pseudodifferential Operators 1968 (Edizioni Cremonese, Roma, 1969), pp. 169-305.

B. BOOSS-BAVNBEK, IMFUFA, Department of Sciences, Systems and Models, Roskilde University, Postboks 260, DK-4000 Roskilde, Denmark

e-mail: booss@ruc.dk 